\title{
25 Research Soure \\ Vaccination Against Influenza Among Lebanese Health Care Workers in the Era of Coronavirus Disease 2019
}

Dalal Youssef ( $\square$ Dalalyoussef.esu@gmail.com )

Ministry of Public Health

Linda Abou Abass

Ministry of Public Health

Janet Youssef

Zahraa University Medical Center

Atika Berry

Ministry of Public Health

\section{Research Article}

Keywords: Health care workers, influenza, vaccination, Coronavirus disease 2019, knowledge, attitudes, practices

Posted Date: February 8th, 2021

DOI: https://doi.org/10.21203/rs.3.rs-150253/v1

License: (c) (i) This work is licensed under a Creative Commons Attribution 4.0 International License. Read Full License 


\section{Abstract}

Background: Health care workers (HCWs) are at increased risk of influenza infection. Hence, the rationale for immunization to protect them and their patients particularly during the co-circulation of influenza and coronavirus disease 2019 (COVID-19). This study aims to assess knowledge, attitudes, and practices among HCWs towards the influenza vaccine and to identify the determinants of their willingness to vaccinate.

Methods: Using an online survey, a cross-sectional study, was conducted in Lebanon between 14th and 28th October 2020 among HCWs. Descriptive statistics were reported using frequency and percentages for categorical variables. Multivariable logistic regression was carried out to identify the factors associated with influenza vaccine uptake. Adjusted odds ratio and their $95 \%$ confidence intervals were reported.

Results: A total of $560 \mathrm{HCWs}$ participated in the survey of which $72.9 \%$ were females. The majority were nurses (63.2\%), married (66.1\%) and aged between 30-49 years (53.9\%). Nearly half of them had a good level of knowledge $(\geq 80 \%)$. As perceived by HCWs, the availability of a sufficient quantity of vaccine will be the biggest challenge. Benefits of vaccination in enhancing patient safety, and avoiding co-infection by influenza and COVID-19 were wellacknowledged by HCWs. $32.1 \%$ of HCWs have received the influenza vaccine during the last year and $80.2 \%$ were willing to vaccinate for the current year. The influenza uptake was higher among HCWs who have received influenza vaccine in the past season $(\mathrm{OR}=6.812, \mathrm{Cl}(3.045-15.239))$, having a fair health status $(\mathrm{OR}=3.117, \mathrm{Cl}(1.345-$ 7.220)), good knowledge (OR=3.305, $\mathrm{Cl}(1.155-9.457)$ ), positive attitude (OR=2.305, $\mathrm{Cl}(0.921-5.571)$ ), low perception of barriers $(\mathrm{OR}=4.130, \mathrm{Cl}(1.827-9.334))$ and high perception of benefits $(\mathrm{OR}=6.264, \mathrm{Cl}(2.919-13.442)$ and was lower in single and divorced (OR=0.527, $\mathrm{Cl}(0.284-0.978))$.

\section{Conclusion}

Addressing misconceptions unveiled in this study through adapted training, along with economic interventions are needed to improve influenza vaccination coverage among HCWs.

\section{Introduction}

Influenza is a contagious respiratory tract disease that represents a foremost global public health challenge. [1] This debilitating illness spreads mainly by droplets and leads to substantial morbidity and potential fatalities yearly [2]. Three varieties of influenza viruses overtly influenza A, B and C exist. In particular, types A and B can cause seasonal epidemics in humans specifically influenza $A$ [3]. Cases infected by influenza are often mild and characterized by fever associated with respiratory as well as systemic symptoms such as muscle or body aches, headache, and fatigue. However, the course of influenza can be severe, since some cases can require hospitalization and admission to the intensive care unit [4]. The severity of influenza among cases in any given year depends on several factors and conditions including age and comorbidities and reflects the degree of genetic drift or shift in the dominant strain of the influenza virus as well as the efficacy and coverage of vaccination [5]. Higher morbidity and mortality rates are noticed particularly in high-risk groups including infants, elderly people, pregnant women, individuals with underlying medical conditions, and health care workers (HCWs) [6].

Bearing in mind, this year the word will face a bigger challenge: seasonal influenza that is still not copiously preventable perplexed by a second respiratory virus that topmost the coronavirus disease 2019 (COVID-19) pandemic [7]. The COVID-19 virus is associated with several-fold higher morbidity and mortality than that of influenza [8]. To be noted that COVID-19 widespread is owed to the naïve immunity among people and the absence 
of COVID-19 vaccine. In fact, the severe acute respiratory syndrome coronavirus 2 (SARS-COV2) that causes COVID19 , and the influenza virus are considerably different pathogens, but there are important areas of overlap. Both viruses are primarily transmitted by respiratory droplets but no specific clinical manifestations reliably extricate early influenza disease and COVID-19. It is almost certain that influenza strains will circulate in conjunction with COVID-19, it is now known that these two viruses can co-infect the same patient [9].

Given their occupational duties and their working environment, HCWs are not solely susceptible to catch influenza and COVID-19, but also embody a noteworthy root of transmission of the viruses to patients under their care as well as circulation of viruses within the community[10]. Such nosocomial transmission has the potential to induct an outbreak within the health care facility. The risk of transmission rises when HCWs continue to perform their duties after falling sick. In fact, HCWs in practices often use non-pharmacological interventions (NPI) as protective measures during the influenza season [11]. However, influenza vaccination is considered the backbone for extenuating influenza outbreaks and disease severity, especially in high-risk groups. It is an effective infection control strategy. For instance, influenza vaccines are known to be $60 \%$ protective among healthy people and decrease illness duration and severity in symptomatic individuals [12]. In recognition of this fact, health institutions such as the World Health Organization (WHO) and the Strategic Advisory Group of Experts (SAGE) on Immunization recommend the prioritization of HCWs vaccination against seasonal influenza[13,14]. Evidence suggests that the vaccination of HCWs may efficiently reduce employee absenteeism triggered by influenza. Therefore, the role of influenza vaccination does not only protect HCWs and thus contribute to maximizing their productivity, but it also avoids patients from becoming sicker by providing additional protection by herd immunity and by improving patient safety. Moreover, in the era of COVID-19, vaccination against influenza would be important to mitigate these infections by decreasing hospital stays due to complications $[15,16]$. Despite such public health interventions and the availability of guidelines, influenza vaccination coverage among HCWs around the world continues to be low. Reasons for the low uptake of influenza vaccine among HCWs are the unavailability of the vaccine, misconception of vaccine safety and effectiveness, and inadequate knowledge about the disease [17].

In Lebanon, the influenza vaccine is not mandatory neither a captivating part of the national vaccination program. Besides, out-of-pocket vaccine expenses are paid by the population; even vaccines are not distributed for free of charge to the vulnerable groups [18]. However, a memo number 149 issued by the ministry of public health on October 6th, 2020 recommends the yearly influenza vaccination of HCWs and designate the target groups to be prioritized for vaccination. A study conducted by Romani et al. evaluating the knowledge and beliefs of Lebanese family physicians regarding influenza vaccines reported a lack of vaccination awareness and poor practices [19]. Overall, there is a scarcity of data related to the influenza vaccine uptake, knowledge, and attitudes among HCWs towards it. Identifying the factors responsible for low vaccination coverage will contribute to developing effective interventions that will help increase influenza vaccine uptake among HCWs. In light of this, examining vaccination coverage rates in the era of COVID-19 as well as understanding the beliefs of HCWs towards influenza vaccine may be an essential first step to suggesting recommendations on vaccination practices and to aid regulatory bodies to implement effective interventions. This would raise the rate of influenza vaccination among HCWS and alleviate the hospital and other health systems the significant pressure of dealing with the conjunction of circulation of COVID19 and influenza in the same period.

The objectives of the present study are to evaluate the seasonal influenza vaccination rate in the era of COVID-19 as well as to assess the knowledge, attitudes, and practices (KAP) among HCWs towards the seasonal influenza vaccine. Besides, this survey presented the opportunity to identify which determinants were associated with influenza vaccine uptake among HCWs. 


\section{Methods}

\section{Study tool and design}

A cross-sectional study, using an online survey, was conducted during the early phase preceding the starting of the influenza seasonal epidemic in Lebanon between 14th and 28th October 2020. As the Lebanese government recommended the public to minimize face-to-face interaction, potential respondents were electronically invited to participate.

\section{Instrumentation}

An extensive review of the literature was conducted to identify relevant items on KAP of influenza vaccination among HCWs. A structured questionnaire was developed by the authors and then reviewed by a panel of 4 experts that included a medical doctor, epidemiologist, infectious diseases expert, and a hygienist. The experts were asked to evaluate the content validity index based on the relevance, coverage, and representativeness of the items in assessing the KAP of HCWs towards vaccination. The CVI was calculated for each item and three of them were rated irrelevant, thus they were omitted from the questionnaire. Then, the drafted version was translated and adapted to the Arabic language. [20]. A pilot study was performed on $10 \mathrm{HCWs}$ to evaluate the comprehensibility and clarity of the items. Minor linguistic edits were made. A final version of the questionnaire was produced and consisted of four sections: (1) Sociodemographic characteristics of the participants; (2) knowledge of influenza and its vaccine (3) attitudes towards influenza vaccine; and (4) practices towards Covid-19.

Socio-demographic information including age, gender, marital status, urbanicity, specialty, place of work, type of hospital, clinical experience, health status, underlying conditions, and health coverage. Participants were also asked whether they have received the influenza vaccine in the past season and if they have refused any kind of vaccination previously.

Knowledge section: Six domains were designed to assess HCW knowledge toward Influenza vaccination: Nature, symptoms, and transmission of influenza (6 items), overlapping between COVID-19 and influenza (3 items), vaccine Effectiveness \& Safety (5 items), Administration, Storage \& Handling (3 items), target Groups for Vaccination (4 questions) and Timing of vaccination (3 items). All the items, except item K15, were answered on a true/false and "do not know" option. A correct response was given a value of ' 1 ' and a "wrong" or "don't know" response was assigned a value of ' 0 '. The item K15 exploring the prioritized groups for influenza vaccination was in the form of multiple choice question in which a value of 1 was assigned for correct answer and 0 for incorrect or unknown answers. Hence, an overall knowledge score would range from 0 to 28 points. HCWs were categorized as having good knowledge if the score was between 80 and 100\% (22-28 points), moderate if the score was between 50 and $79 \%$ (14-21 points), and poor if the score was less than $50 \%$ (< 14 points).

Attitudes section: Two dimensions with a total of 15 items were used to assess HCW attitudes toward influenza and vaccination (9 items) and toward health facility, government and society (6 items). Responses to questions related to attitude were graded on a 5-point Likert scale, an agreement scale ranging from ' 1 ' for strongly disagree to ' 5 ' strongly agree. A point of 1 was given to the 2 options "strongly agree" and "agree" answer while strongly disagree, disagree or neutral responses were given a 0 point. The overall level of attitude was categorized using original Bloom's cut-off point, as positive if the score was $80-100 \%$ (12-15 points), neutral if the score was $50-$ $79 \%$ (8-11 points) and negative if the score was less than $50 \%$ (< 8 points). 
Perceived barriers and benefits section: this part comprises 7 items concerning the main barriers and 5 items reflecting benefits of influenza vaccination as perceived by HCWs.

Practice section: Two main domains constitute this section. The first one was about the uptake of influenza vaccination and it was based on only one question regarding willingness to vaccinate during the current influenza season. The item was answered "yes" and "no". The answer "yes" was assigned 1 point whereas "no" answer was assigned 0 points. The second domain was about practices and behaviors related to vaccine promotion and continuous education (training). Thirteen questions were used to evaluate positive behaviors.

The questionnaire was validated by doing a pre-test on $5 \%$ of the sample before the actual data collection period. Survey flow, functionality, and language were based on pre-test feedback. Furthermore, the reliability of the questionnaire was checked, and their Cronbach Alpha value was 0.82 . The average time for filling the survey was 8 minutes.

\section{Sample size calculation}

To calculate the sample size of the study, the Raosoft sample size calculator designed specifically for population surveys was used. Assuming that between 50000 registered HCWs, 40000 of them are actively practicing at the health facilities level, a 95\% confidence level was used and an absolute error was estimated to be $5 \%$. All previous information was used to calculate the sample size for this study which yielded the least required sample size of 381 participants. The required sample size was achieved one week before the closure of response acceptance (October $\left.28^{\text {th }}, 2020\right)$.

\section{Data collection}

An online questionnaire using a Google form was emailed to governmental run and private hospital directors to ensure officialism. Then, designated focal persons working in Lebanese hospitals were contacted via phone call and notified about the survey and its purpose. Upon their agreement to participate, the link of the study was sent through "WhatsApp" to the designated focal person (infection control personnel) who was requested in his turn to diffuse it among other facility HWCs using a snowball technique. This link includes a brief introduction to the background, the objective of the survey, and instructions for filling the questionnaire.

Participants were identified via the infection control personnel at the hospital. All HWCs, working in Lebanese hospitals in different provinces in Lebanon and who agreed to participate in the study, were eligible for participation. The administrative staff was not included in this survey. All methods were carried out in accordance with relevant guidelines and regulations [21].

A written informed consent was obtained for every participant. They were reassured that their participation is voluntary and that they were free to withdraw at any time, without giving a reason and without cost. In addition, all information were gathered anonymously and handled confidentially. The questionnaire was collected only in subjects who expressed consent for study participation. As individual participants cannot be identified based on the presented material, this study caused no plausible harm or stigma to participants. The study design assured adequate protection of study participants, and neither include clinical data about patients nor configure itself as a clinical trial. Hence, this study is exempt from ethical approval in the ministry of Public Health.

\section{Data analysis:}


All the statistical analyses were performed using the statistical software SPSS (Statistical Package for Social Sciences), version 22.0. Categorical variables were expressed using frequencies and percentages. Chi-square test was used to determine the associations between influenza uptake and independent variables including demographic characteristics, perceived barriers and benefits as well as KAP scores. Multivariable logistic regression was also carried on the significant variables in the bivariate analyses/chi-squared test with $p$-value $<0.2$ to identify the predictors of influenza uptake among HCWs. For all tests, $P$ values $<0.05$ were considered as statistically significant.

\section{Results}

\section{Baseline characteristics of the study participants}

Table 1 shows the baseline characteristics of the participants. A total of $560 \mathrm{HCWs}$ participated in the survey of which $72.9 \%$ were females. About two-third were married (66.1\%) and approximately half of them (53.9\%) were aged between $30-49$ years. The majority of them were nurses (63.2\%). Of the total, $75.4 \%$ of participating HCWs are working mainly in private settings. Regarding their work experience, $43.8 \%$ of the participants had a large work experience (more than 10 years of experience). Moreover, $85 \%$ of them reported good health status and the majority are health covered by public insurance (87.5\%). It is noteworthy, that only $32 \%$ of HCWs have received their influenza vaccine in the past influenza season and $14.6 \%$ of them reported refusal of a certain type of vaccine in the past.

\section{HCWs self-reported knowledge}

Nearly half of the HCWs (47.9\%) had a good level of knowledge ( $\geq 80 \%)$ and less than $1 \%$ of HCWs had an overall poor level of knowledge. Table 2 illustrates the knowledge about vaccination against novel Coronavirus among the HCWs. The majority of the respondents were aware of the nature, symptoms and, transmission of influenza (70\%), the importance and the safety of the influenza vaccine (77\%) as well as the timing of vaccination (71.5\%). Knowledge was also good for the overlapping of two respiratory viruses (COVID-19 and influenza) domain (70\%). Poor knowledge was more apparent in response to questions related to the awareness regarding the influenza vaccine target groups (30\%).

Table 3 describes HCWs answers towards influenza vaccine knowledge items. Despite the good level of knowledge recorded in the domain related to the nature and transmission of influenza, almost $49.5 \%$ of HCWs were not knowledgeable that symptoms appear 8 to 10 after exposure to the influenza virus. In addition, $23.8 \%$ of them didn't recognize that influenza vaccine can continue to be offered as long as influenza viruses are circulating. The unveiled poor knowledge in the vaccine target groups domain shown in table 2 was particularly related to the question concerning the prioritization of target groups when a limited supply of vaccine is experienced, where more than $60 \%$ of HCWs didn't consider children aged 6 months through 4 years (59 months) and pregnant woman as a priority for immunization in such situation.

\section{HCWs attitudes:}

\subsection{Assessment of HCWs attitudes towards influenza vaccination}

Table 3 describes HCW's attitude towards influenza vaccination. The majority disagree about considering influenza vaccine as facultative (75.1\%) and about sighting influenza as a mild disease not necessitating vaccination against 
(80.9\%). Almost half (53.6\%) of the participants disagree that benefits of avoiding the disease are not enough and that healthy people do not need vaccination (61.2.\%) while, only $9.1 \%$ of HCWs agreed that vaccines weaken or overload the immune system and that allergies are on rise because of vaccination (12.8\%). Of the total HCWs, only $22.8 \%$ agreed that is better to develop natural immunity rather than getting vaccinated. It is worth noting that although that $64.2 \%$ of them consider vaccines among the safest and most tested medicinal products only $40 \%$ agreed that the adverse reactions resulting from influenza vaccination were underestimated.

\subsection{HCWs' attitudes towards health facility, health authorities and, societies}

Out of $560,327(58.4 \%)$ consider that Lebanese society has more important problem than influenza. The majority of them $(85.6 \%)$ declared that the government should finance the vaccine for all and that vaccine policy in Lebanon is influenced by the financial profits of pharmaceutical companies (63.3\%). More than $40 \%$ of HCWs consider that the health facility where they work should ensure the availability of influenza vaccines at their institution. Whereas, approximately half of the participants (52.5\%) considered that information provided by health authorities is reliable and $33 \%$ of them were neutral regarding this issue (Table 4 ).

\subsection{Barriers for influenza vaccination:}

Figure 1 presents the perceived barriers for influenza vaccination. The majority of HCWs were not concerned about the mainstream barriers. In the light of COVID-19, $83.3 \%$ of the HCWs considered that the biggest challenge will be the availability of a sufficient quantity of vaccine and the conceivable shortage faced due to the high demand during the current year. The fear of development of a severe adverse event was stated as a barrier by $31.07 \%$ of HCWS followed by the cost (24.29\%) and the concern about side effects resulting from vaccination (23.57\%). Approximately $18.21 \%$ of HCWs were concerned about influenza vaccine safety. A slight proportion of HCWs (7.86\%) assumed that the influenza vaccine is not effective and only $4.86 \%$ of them showed concern due to the fear from the needle.

\subsection{HCWs' s perceived benefits of influenza vaccination:}

Figure 2 shows the benefits of influenza vaccination perceived by HCWs. The majority of HCWs thought that their influenza vaccination can enhance patient safety $(86.43 \%)$. In addition, they believed that it can minimize the viral reservoir in the population (82.5\%), decrease hospital visits (81.25\%) and avoid co-infection by influenza and COVID-19 (78.75\%) allowing consequently health services to better cope with COVID-19 complications. Only 39.29\% of HCWs consider influenza vaccination cost-effective.

\section{HCWs practices toward influenza vaccination}

\subsection{Part 1: Influenza uptake}

Of the total HCWs, only one third (32.1\%) declared their uptake of the influenza vaccine the last year. However, the majority of HCWs (80.2\%) declared their willingness to uptake influenza vaccine the current year. Thus, an outstanding increase of almost $50 \%$ in the proportion of vaccinated HWCs is clearly shown. (Figure 3 )

\subsection{Part 2: Other practices of HCWs}

\subsubsection{Promotion and advertising of influenza vaccine}


Table 5 summarizes the positive behaviors reported by HCWs regarding vaccine promotion and training. More than $50 \%$ of HCWs declared that they always encourage their social and working environment to get vaccinated (patients, colleagues, family members). Around $30 \%$ of them promote occasionally vaccine uptake and less than $10 \%$ of them reported that they never encourage influenza vaccination regardless of the target categories. About $40.5 \%$ of HCWs always communicate the importance of vaccination during hospital and clinic visits and $43.4 \%$ of them do it occasionally. For the sake of avoiding dual infection by COVID-19, approximately $92.7 \%$ of HCWs encourage flu vaccine whether doing it always (61.1\%) or occasionally (31.6\%). However, the use of phone and email as tools to promote the importance of influenza vaccination was less reported among HCWs. Nearly half of HCWs declared that they never use this option (mobile, email...) or celebrate events related to influenza vaccination (vaccine day). Similarly, the majority of HCWs (53.2\%) didn't send vaccine reminders to patients. However, more than $70 \%$ of HCWs recourse to use always $(23.8 \%)$ or occasionally $(47.1 \%)$ printing materials like brochures and posters to elucidate the importance of vaccination.

\subsubsection{Trainings and workshops:}

As showed in table 5, the majority of HCWs (91.8\%) have attended training about vaccination in the past and only $8.2 \%$ have never joined any training focusing on influenza. Of the total, $46.3 \%$ of HCWs always encourage other staff and colleagues to attend such conferences and training.

\section{Factors associated with influenza uptake}

Table 6 represents the multivariable logistic regression of the factors associated with influenza uptake. Our results showed that the odds of influenza uptake practice was 6.812 times higher among HCWs who have received influenza vaccine in the past season compared to those who were not vaccinated in the last season (Adjusted OR = 6.812 with $95 \% \mathrm{Cl}$ of 3.045 to 15.239 ). In opposite, the vaccination uptake was lower in single and divorced HCWs than their counterparts who are married (adjusted OR=0.527 with $95 \% \mathrm{Cl} 0.284$ to 0.978 ). HCWs who declared that their health status is fair or below were 3.117 more likely to get influenza vaccination compared to HCWs with good or above health status (adjusted OR $=3.117$ with $95 \% \mathrm{Cl}$ of $1.345-7.220$ ). Similarly, respondents with positive attitude towards influenza vaccine were 2.305 more likely to uptake influenza vaccine (OR=2.305 with $95 \% \mathrm{Cl} 0.921$ 5.571). Furthermore, the influenza vaccine uptake was 4.130 higher among HCWs who had low perception of barriers comparing to those with high perception of barriers. With regards to perceived benefit, HCWs who had high and moderate perception level of benefits were more likely to get vaccinated than their counterparts with low perception of benefits (OR=6.264 with $95 \% \mathrm{Cl} 2.919$ to 13.442). Finally, participants with good knowledge toward the safety of vaccine were 3.305 times more conceivable to get the vaccine (OR=3.305, with $95 \% \mathrm{Cl} 1.155$ to 9.457 ) than other HCWs with poor knowledge regarding this domain.

\section{Discussion}

To the best of our knowledge, this study is the first in Lebanon to evaluate the influenza uptake rate among hospital-based HCWs in the context of COVID-19 and to determine factors associated with the willingness to vaccinate by exploring knowledge, attitudes, perceived barriers, benefits, and practices among this population. The study's main findings are that almost all surveyed HCWs have a sufficient knowledge level with nearly half of them had a good knowledge level ( $\geq 80 \%)$. These results are consistent with the findings displayed in a study conducted in the United States reporting a knowledge level $\geq 80 \%$ among nurses [22]. Besides, less than $1 \%$ of HCWs exhibited a poor overall knowledge score. The gaps in knowledge were more ostensible and concrete in the knowledge 
domain related to the prioritization of the target groups when a limited supply of vaccine is experienced. A peculiar finding in this study was that more than half of surveyed HCWs considered that pregnant women are not among prioritized groups for influenza vaccination. Lack of consciousness among HCWs about the importance of influenza vaccination among pregnant women could lead them to skip advising pregnant women about the essentiality of influenza vaccine during pregnancy. Many studies reflected that lack of knowledge among health care providers (HCP) could impact negatively the pregnant women uptake of influenza vaccine since their decision was based basically on compelling advice provided by HCWs [23-25].

Moreover, $60 \%$ of surveyed HCWs didn't believe that children aged between 6 months and 5 years are among the prioritized target groups for influenza vaccination. Many studies shed light on the importance of prioritizing influenza vaccination of children to minimize infection in the community and because they had the longest lives ahead of them $[14,26,27]$.

Another important finding in this study was that nearly half of HCWs were not aware about the incubation period of the influenza virus and $23.8 \%$ of them didn't recognize the timeframe of influenza vaccination and the fact the vaccine can continue to be offered as long as influenza viruses are circulating (timeframe). Such deficiency in knowledge concerning target groups and timing of vaccination could lead to the falloff of the vaccination coverage among HCWs and the Lebanese population. These gaps in knowledge underline the importance of raising awareness among HCWs regarding influenza target groups and vaccination timeframe.

The majority of HCWs considered influenza as a serious disease and they support that vaccination against influenza should be mandatory. A study conducted in Saudi Arabia exhibited that recommendations from the government on influenza vaccination for HCWs and compulsory requirements were significant determinants of vaccine acceptance [28].

Almost half of them disclosed that even healthy people do need to vaccinate and that benefits of evading the disease are pertinent. However, a critical review conducted by Abramson showed that the personal benefit from vaccinating healthy nonelderly adults is small [29]. Besides, the majority of HCWs disagreed about the ability of vaccines to weaken or overload the immune system. They disapproved that influenza vaccination is leading to the rise of allergies as well. To date, utmost studies disclosed strong evidence that no association between influenza vaccines and increased risk for atopy, asthma symptoms, exacerbations, or increased use of rescue medications $[30,31]$.

However, $28.8 \%$ of them thought that developing natural immunity is better than getting the vaccine. Despite the fact, that a flu infection would stimulate the immune system more strongly than the vaccine will, but that comes with a substantial price in case that the person got sick and developed complications and might die. In addition, the immunity gained from a bout of influenza only protects against a particular subtype but that's just one of the four lineages, and it drifts and won't last long because of those rapid mutations of the influenza virus [32]. Besides, more than one-third of HCWs thought that adverse reactions resulting from influenza vaccination were underestimated. These results are consistent with the findings of a study conducted in Germany where physicians and nonvaccinated nurses overestimated the rates of both non-severe and severe adverse effects. This was negatively associated to vaccination rates among all professionals [33].

Concerning their attitudes towards the Lebanese government, more than half of HCWs declared that the Lebanese society has more vital challenges and trials further than influenza. This could be due to the heavy economic crisis, 
accompanied by a dangerous depletion of resources, including human capital aggravated by the COVID-19 pandemic and the Beirut blast.

The majority of HCWs also considered that influenza vaccine would be provided for free and should be financed by the government and more than $40 \%$ of them thought that the health facility where they work should ensure the availability of influenza vaccine at their institution. In some neighboring countries, influenza vaccines are offered free of charge for all the population [34]. Despite that influenza vaccine is not considered costly, but, ensuring its availability at the health facility and providing it for free could increase vaccination coverage among health care workers

In addition, some HCWs appraised that vaccine policy in Lebanon is influenced by the financial profits of pharmaceutical companies. This could be well understood given the Lebanese loss of trust toward the political system. Despite this fact, nearly half of HCWs ponder that information provided by health authorities is reliable.

Another noteworthy finding in the study is that $83.3 \%$ of the HCWs listed the availability of a sufficient quantity of vaccine for the current year at the top of challenges that will hinder their access to vaccines. The conceivable shortage faced will be due to the high demand during the current year. The panic generated by the COVID-19 pandemic and the fear of getting co-infected has prompted people to seek influenza vaccination, in order to prevent COVID-19 complications and reduce the burden on the healthcare system.

In addition, the development of side effects (23.57\%), severe adverse events (31.07\%), and vaccine safety (18.21\%) were listed between impediments of vaccination. Since the cost of the vaccine is paid out-of-the-pocket in Lebanon, the cost of the vaccine was stated as a barrier. Similar impediments of vaccination were revealed in different studies conducted in Singapore and Saudi Arabia [35-37].

The main benefits mentioned by HCWs' uptake or willingness to be vaccinated include enhancing patient safety, minimizing the viral reservoir in the population, decreasing hospital admission due to influenza, and avoiding coinfection by influenza and COVID-19 allowing consequently health services to better cope with COVID-19 complications. Whereas, only $39.29 \%$ of HCWs ponder influenza vaccination as a cost-effective intervention. Our results are consistent with the benefits reported in many studies. A systematic review of randomized trials showed that HCWs' influenza vaccination can enhance patient safety $[9,38,39]$.

Despite the annual recommendation of influenza vaccination issued by the ministry of public health, the findings of our study showed that only $32.1 \%$ of the surveyed HCWs received their influenza vaccine in the past season (20192020). This vaccination coverage rate is considered low compared to the neighboring countries. A recent review reported that the lowest rate of HCWs' vaccination against influenza was recorded in Vietnam (3.7\%) and the highest one was accorded to Japan (85.7\%) [40]. For the season 2016-2017, Qatar reached a vaccination coverage rate of $77 \%$ among HCWs [41]. Similarly, almost $67.6 \%$ of HCWs were vaccinated in the 2016 season in Saudi Arabia [28]. Indeed, a higher influenza vaccination rate could be attributed to the fact that these countries have ensured the availability of free of charge vaccines and conduct vaccination campaigns.

One noteworthy finding in the present study is that uptake of influenza vaccine during this season (2020-2021) which overlaps with the circulation of SARS-Cov2, rise to $80.2 \%$ among HCWs. Given that medical professionals stressed the importance of the influenza vaccine during the COVID-19 pandemic, the willingness to vaccinate against influenza in the current year seemed to be highly affected by the COVID-19 pandemic. In addition, receiving the influenza vaccine in the past season is highly associated with the willingness to vaccinate during the current

Page 10/25 
season. Similar results were found by a study conducted in Germany where receiving the influenza vaccination in the previous season was the strongest independent predictor of pandemic influenza vaccination [42]. These findings also were reflected in many systematic reviews that have repeatedly recognized past behavior as a strong predictor of influenza vaccine acceptance $[43,44]$.

Regarding marital status, we found that being separated or divorced decreased the vaccination uptake. This result is consistent with the findings of the study that found that being widowed increased the decision to become immunized while being separated or divorced decreased it [45]. Despite some authors found no relationship between those variables, marital status is considered an important predictor of healthcare utilization. In fact, marriage may influence health status not only through the support and protection that marriage offers but also through a more efficient pattern of healthcare utilization [46].

In this study, the large experience of HCWs was not linked to the intention to receive vaccine despite that other studies have associated large experience to high willingness and acceptance rate. Similar findings were observed in a study conducted in Oman where experience did not correlate with greater compliance with vaccination [47]. In addition, HCWs who rated their health status as fair or below were more likely to get influenza vaccination compared to other HCWs who had a good health status. Similar findings were reported in a study conducted among Italian HCWs [48].

On the contrary, the professional occupation was not associated with vaccine uptake. No significant difference was shown between physicians, nurses, laboratory technicians, and pharmacists. However, a study conducted in Dubai disclosed an association between the professional occupation of HCWs and vaccines with the highest uptake among physicians followed by nurses. Less perception of barriers and positive perception of benefits were associated with a high willingness to vaccinate. Our results disclosed also that a good knowledge score was associated with the willingness to be immunized against influenza. Participants with good knowledge of the safety of vaccines were more conceivable to get the vaccine. These findings were in line with the results displayed in a study conducted in Dubai where the proportion of HCWs with good knowledge had a higher level of acceptance of vaccine [49].

The adoption of positive behaviors such as encouraging patients, relatives, and colleagues to get vaccinated against influenza, attending training related to vaccination, and addressing influenza vaccine hesitancy was reported among HCWs. Many studies highlighted the importance of positive behavior in improving the vaccination coverage rate.

\section{Limitations Of The Study}

Some limitations of this study should be acknowledged. First, our study relies on HCWs self-reported information, which may be a threat to information bias. In addition, self-reported information could be associated with recall bias about past behaviors. On other hand, participants responses may be influenced by social desirability and then intendedly modified to meet the norms. Furthermore, this survey was conducted during the earlier phase of influenza season, therefore, the attitudes and practices reflected only the information available at that time.

\section{Conclusion}

Despite these limitations, the present study provides useful insights on the knowledge, attitudes and practices of HCWs on influenza. Promoting vaccine uptake among HCWs is a core component of patient and HCWs safety. 
Programs that actively target identified gaps and barriers identified in this study are recommended. Improving access to vaccination, addressing common misconceptions through adapted HCWs training and education based on the unveiled gaps. Besides individual level behavior change, economic related interventions may be considered like compulsory influenza vaccination and free of charge vaccine within health facilities which would increase and sustain high influenza vaccination rate.

\section{Declarations}

\section{Ethics approval and consent to participate}

The study design respected the participant's confidentiality and autonomy. A written informed consent was obtained from all participants. As survey studies are exempt from ethical approval in Lebanon according to national regulations [50], the Lebanese Ministry of Public Health waived the need for ethical approval.

\section{Authors' contributions}

DY developed the project idea. DY, JY and LAA formulated the questionnaire, organized and analyzed the survey. $D Y, J Y, L A A, A B$ drafted and critically reviewed the paper. All authors read and agreed on the final version.

\section{Funding}

No funding was received.

\section{Availability of data and materials}

Data are available from the corresponding authors upon reasonable request.

\section{Consent for publication}

Not applicable.

\section{Competing interests}

The author(s) declare that they have no competing interests.

\section{Acknowledgments}

We would like to acknowledge all health care workers participating in the study

\section{Authors information}

Dalal Youssef, MPH, PHD candidate, Ministry of public Health, Lebanon, Preventive medicine department 
Atika Berry, MD, Ministry of Public Health, Preventive medicine department

Janet Youssef, MD, OBGYN, ZHUMC

Linda Abou Abbas, MPH, PHD, Ministry of Public Health, Epidemiological surveillance unit

\section{References}

1. Ortiz, J.R., et al., Strategy to enhance influenza surveillance worldwide. Emerging infectious diseases, 2009. 15(8): p. 1271-1278.

2. Moghadami, M., A Narrative Review of Influenza: A Seasonal and Pandemic Disease. Iranian journal of medical sciences, 2017. 42(1): p. 2-13.

3. Arbeitskreis Blut, U.B.B.K., Influenza Virus. Transfusion medicine and hemotherapy : offizielles Organ der Deutschen Gesellschaft fur Transfusionsmedizin und Immunhamatologie, 2009. 36(1): p. 32-39.

4. Caini, S., et al., Clinical characteristics and severity of influenza infections by virus type, subtype, and lineage: A systematic literature review. Influenza and other respiratory viruses, 2018. 12(6): p. 780-792.

5. Martínez, A., et al., Risk factors associated with severe outcomes in adult hospitalized patients according to influenza type and subtype. Plos one, 2019. 14(1): p. e0210353-e0210353.

6. Restivo, V., et al., Influenza vaccine effectiveness among high-risk groups: A systematic literature review and meta-analysis of case-control and cohort studies. Human vaccines \& immunotherapeutics, 2018. 14(3): p. 724735.

7. Belongia, E. and M. Osterholm, COVID-19 and flu, a perfect storm. Science, 2020. 368: p. 1163-1163.

8. Alwan, N.A., et al., Scientific consensus on the COVID-19 pandemic: we need to act now. Lancet, 2020. 396(10260): p. e71-e72.

9. Solomon, D.A., A.C. Sherman, and S. Kanjilal, Influenza in the COVID-19 Era. JAMA, 2020. 324(13): p. 13421343.

10. Maltezou, H.C. and A. Tsakris, Vaccination of health-care workers against influenza: our obligation to protect patients. Influenza and other respiratory viruses, 2011. 5(6): p. 382-388.

11. Smith, S.M.S., et al., Use of non-pharmaceutical interventions to reduce the transmission of influenza in adults: A systematic review. Respirology (Carlton, Vic.), 2015. 20(6): p. 896-903.

12. DiazGranados, C.A., M. Denis, and S. Plotkin, Seasonal influenza vaccine efficacy and its determinants in children and non-elderly adults: A systematic review with meta-analyses of controlled trials. Vaccine, 2012. 31(1): p. 49-57.

13. Meeting of the Strategic Advisory Group of Experts on immunization, April 2012--conclusions and recommendations. Wkly Epidemiol Rec, 2012. 87(21): p. 201-16.

14. Zarocostas, J., Healthcare workers should get top priority for vaccination against A/H1N1 flu, WHO says. BMJ (Clinical research ed.), 2009. 339: p. b2877.

15. Anikeeva, O., A. Braunack-Mayer, and W. Rogers, Requiring influenza vaccination for health care workers. American journal of public health, 2009. 99(1): p. 24-29.

16. Cortes-Penfield, N., Mandatory influenza vaccination for health care workers as the new standard of care: a matter of patient safety and nonmaleficent practice. American journal of public health, 2014. 104(11): p. 20602065. 
17. Perl, T.M. and T.R. Talbot, Universal Influenza Vaccination Among Healthcare Personnel: Yes We Should. Open Forum Infectious Diseases, 2019. 6(4).

18. El Khoury, G. and P. Salameh, Influenza Vaccination: A Cross-Sectional Survey of Knowledge, Attitude and Practices among the Lebanese Adult Population. Int J Environ Res Public Health, 2015. 12(12): p. 15486-97.

19. Romani, M.H., et al., Family physicians beliefs and attitudes regarding adult pneumococcal and influenza immunization in Lebanon. Fam Pract, 2011. 28(6): p. 632-7.

20. Tsang, S., C.F. Royse, and A.S. Terkawi, Guidelines for developing, translating, and validating a questionnaire in perioperative and pain medicine. Saudi journal of anaesthesia, 2017. 11(Suppl 1): p. S80-S89.

21. Kaliyaperumal, K., Guideline for conducting a knowledge, attitude and practice (KAP) study. AECS Illumination, 2004. 4: p. 7-9.

22. Ofstead, C.L., et al., Influenza vaccination among registered nurses: information receipt, knowledge, and decision-making at an institution with a multifaceted educational program. Infect Control Hosp Epidemiol, 2008. 29(2): p. 99-106.

23. Buchy, P., et al., Vaccinating pregnant women against influenza needs to be a priority for all countries: An expert commentary. International Journal of Infectious Diseases, 2020. 92: p. 1-12.

24. Ishola, D.A., Jr., et al., Midwives' influenza vaccine uptake and their views on vaccination of pregnant women. Journal of Public Health, 2013. 35(4): p. 570-577.

25. Prospero, E., et al., Factors influencing refusing of flu vaccination among pregnant women in Italy: healthcare workers' role. Influenza and Other Respiratory Viruses, 2019. 13(2): p. 201-207.

26. Bambery, B., et al., Influenza Vaccination Strategies Should Target Children. Public health ethics, 2017. 11(2): p. 221-234.

27. Persad, G., A. Wertheimer, and E.J. Emanuel, Principles for allocation of scarce medical interventions. The Lancet, 2009. 373(9661): p. 423-431.

28. Alshammari, T.M., et al., Healthcare professionals' knowledge, attitude and acceptance of influenza vaccination in Saudi Arabia: a multicenter cross-sectional study. BMC Health Services Research, 2019. 19(1): p. 229.

29. Abramson, Z., What, in Fact, Is the Evidence That Vaccinating Healthcare Workers against Seasonal Influenza Protects Their Patients? A Critical Review. International journal of family medicine, 2012. 2012: p. 205464.

30. Miller RL, Cheng M, DiMango EA, Geromanos K, Rothman PB; American Lung Association Asthma Clinical Research Centers. T-cell responses and hypersensitivity to influenza and egg antigens among adults with asthma immunized with the influenza vaccine. J Allergy Clin Immunol. 2003;112(3),606-608.

31. Kmiecik T, Arnoux S, Kobryn A, Gorski P. Influenza vaccination in adults with asthma: safety of an inactivated trivalent influenza vaccine. J Asthma. 2007;44(10), 817-22.

32. Centers for Disease Prevention and Control (CDC). Misconceptions about Seasonal Flu and Flu Vaccines. Available https://www.cdc.gov/flu/prevent/misconceptions.htm. Accessed 7 December 2020.

33. Ehrenstein, B.P., et al., Perceived risks of adverse effects and influenza vaccination: a survey of hospital employees. European Journal of Public Health, 2010. 20(5): p. 495-499.

34. Alqahtani, A.S., et al., Knowledge and attitudes of Saudi populations regarding seasonal influenza vaccination. Journal of Infection and Public Health, 2017. 10(6): p. 897-900.

35. Hwang, S. and H. Lim, Barriers and Motivators of Influenza Vaccination Uptake among Primary Healthcare Workers in Singapore. Proceedings of Singapore Healthcare, 2014. 23: p. 126-133. 
36. Durovic, A., et al., Low rates of influenza vaccination uptake among healthcare workers: Distinguishing barriers between occupational groups. Am J Infect Control, 2020. 48(10): p. 1139-1143.

37. Alsuhaibani, M., Barriers and beliefs among health-care workers regarding seasonal influenza vaccine in AlQassim region, Saudi Arabia. Human Vaccines \& Immunotherapeutics, 2020. 16(2): p. 313-320.

38. Perl, T.M. and T.R. Talbot, Universal Influenza Vaccination Among Healthcare Personnel: Yes We Should. Open forum infectious diseases, 2019. 6(4): p. ofz096-ofz096.

39. Ahmed, F., et al., Effect of Influenza Vaccination of Healthcare Personnel on Morbidity and Mortality Among Patients: Systematic Review and Grading of Evidence. Clinical Infectious Diseases, 2013. 58(1): p. 50-57.

40. Gülten E, Özer Türk D, Erol A, Özel D. The Knowledge, Attitudes and Beliefs of the Healthcare Workers about Influenza Infection and Vaccination at a Public Hospital in Turkey. Infect Dis Clin Microbiol 2019; 2: 87-96.

41. Elawad, K.H., et al., Improving influenza vaccination rate among primary healthcare workers in Qatar. Vaccines, 2017. 5(4): p. 36.

42. Böhmer, M.M., et al., Barriers to pandemic influenza vaccination and uptake of seasonal influenza vaccine in the post-pandemic season in Germany. BMC Public Health, 2012. 12: p. 938.

43. Nagata, J.M., et al., Social determinants of health and seasonal influenza vaccination in adults $\geq 65$ years: a systematic review of qualitative and quantitative data. BMC Public Health, 2013. 13(1): p. 1-25.

44. Prematunge, C., et al., Factors influencing pandemic influenza vaccination of healthcare workers-a systematic review. Vaccine, 2012. 30(32): p. 4733-4743.

45. Portero de la Cruz, S. and J. Cebrino, Trends, Coverage and Influencing Determinants of Influenza Vaccination in the Elderly: A Population-Based National Survey in Spain (2006-2017). Vaccines, 2020. 8(2): p. 327.

46. Pandey, K.R., et al., The impact of marital status on health care utilization among Medicare beneficiaries. Medicine, 2019. 98(12): p. e14871.

47. Awaidy STA, K. Al Mayahi Z, Kaddoura M, Mahomed O, Lahoud N, Abubakar A, Zaraket H. Influenza Vaccination Hesitancy among Healthcare Workers in South Al Batinah Governorate in Oman: A Cross-Sectional Study. Vaccines. 2020; 8(4):661.

48. Barbadoro, P., et al., Determinants of influenza vaccination uptake among Italian healthcare workers. Hum Vaccin Immunother, 2013. 9(4): p. 911-6.

49. AlMarzooqi, L.M., et al., Knowledge, attitude, and practice of influenza vaccine immunization among primary healthcare providers in Dubai health authority, 2016-2017. Human Vaccines \& Immunotherapeutics, 2018. 14(12): p. 2999-3004.

50. Ministry of Public Health, Standards and Operational Guidance for Ethics Review of Health-Related Research with Human Participants,

https://www.moph.gov.Ib/userfiles/files/HealthCareSystem/Pharmaceuticals/ClinicalTrial/WHOGuidelines.pdf, accessed February 1, 2021.

\section{Tables}




\begin{tabular}{|c|c|c|}
\hline & $\mathbf{n}$ & $\%$ \\
\hline \multicolumn{3}{|l|}{ Gender } \\
\hline Male & 152 & $27.10 \%$ \\
\hline Female & 408 & $72.90 \%$ \\
\hline \multicolumn{3}{|l|}{ Age (years) } \\
\hline $20-29$ & 219 & $39.10 \%$ \\
\hline $30-49$ & 302 & $53.90 \%$ \\
\hline$>50$ & 39 & $7 \%$ \\
\hline \multicolumn{3}{|l|}{ Social status } \\
\hline Single & 171 & $30.50 \%$ \\
\hline Married/Engaged & 370 & $66.10 \%$ \\
\hline Divorced & 19 & $3.40 \%$ \\
\hline \multicolumn{3}{|l|}{ Urbanicity } \\
\hline Urban & 314 & $56.10 \%$ \\
\hline Rural & 246 & $43.90 \%$ \\
\hline \multicolumn{3}{|l|}{ Type of hospital } \\
\hline Private & 422 & $75.40 \%$ \\
\hline Public & 138 & $24.60 \%$ \\
\hline \multicolumn{3}{|l|}{ Occupation } \\
\hline Physician & 81 & $14.50 \%$ \\
\hline Lab specialist & 74 & $13.20 \%$ \\
\hline Pharmacist & 51 & $9.10 \%$ \\
\hline Nurse & 354 & $63.20 \%$ \\
\hline \multicolumn{3}{|l|}{ Years of experience } \\
\hline Less than 10 years & 315 & $56.30 \%$ \\
\hline 10 years or more & 245 & $43.80 \%$ \\
\hline \multicolumn{3}{|l|}{ Heath status } \\
\hline Fair or below & 86 & $15.40 \%$ \\
\hline Good or above & 474 & $84.60 \%$ \\
\hline \multicolumn{3}{|c|}{ Presence of comorbidities } \\
\hline Yes & 135 & $24.10 \%$ \\
\hline
\end{tabular}




\begin{tabular}{|lcc|}
\hline No & 425 & $75.90 \%$ \\
\hline Type of hospital & & \\
\hline Private & 422 & $75.40 \%$ \\
\hline Public & 138 & $24.60 \%$ \\
\hline Health Coverage & & \\
\hline Public insurance & 490 & $87.50 \%$ \\
\hline Private insurance & 29 & $5.20 \%$ \\
\hline None & 41 & $7.30 \%$ \\
\hline Received influenza vaccine in the past season & \\
\hline Yes & 180 & $32.10 \%$ \\
\hline No & 380 & $67.90 \%$ \\
\hline Refused vaccination of a certain type of vaccine in the past \\
\hline Yes & 82 & $14.60 \%$ \\
\hline No & 478 & $85.40 \%$ \\
\hline Total & 560 & $100 \%$ \\
\hline
\end{tabular}

Table 2: Knowledge of HCWs towards the seasonal influenza vaccine $(\mathrm{N}=560)$

$\begin{array}{lll}\text { Poor } & \text { Moderate (50\%-79\%) } & \text { Good } \\ (<50 \%) & & (\geq 80 \%) \\ \text { n (\%) } & n(\%) & n(\%)\end{array}$

\section{Domains of Knowledge score}

\begin{tabular}{llll}
\hline Domain 1: Influenza: nature, symptoms and transmission & $52(9.2 \%)$ & $114(20.4 \%)$ & $394(70.4 \%)$ \\
\hline Domain 2: Influenza vaccine importance and safety & $39(7 \%)$ & $90(16 \%)$ & $431(77 \%)$ \\
\hline Domain 3: Influenza vaccine target groups & $\mathbf{1 6 8 ( 3 0 \% )}$ & $107(19.1 \%)$ & $285(50.9 \%)$ \\
\hline Domain 4: Vaccine administration and storage & $74(13.2 \%)$ & $170(30.4 \%)$ & $316(56.4 \%)$ \\
\hline Domain 5: Timing of vaccination & $22(3.9 \%)$ & $138(24.6 \%)$ & $400(71.5 \%)$ \\
\hline Domain 6: Influenza and COVID-19 & $18(3.2 \%)$ & $\mathbf{1 5 0}(26.8 \%)$ & $392(70 \%)$ \\
\hline Knowledge score (28 items) & $\mathbf{2 ( 0 . 4 \% )}$ & $\mathbf{2 9 0 ( 5 1 . 8 \% )}$ & $\mathbf{2 6 8 ( 4 7 . 9 \% )}$ \\
\hline
\end{tabular}


Table 3: Knowledge items $(\mathrm{N}=560)$

$\begin{array}{lll}\# & \text { Correct } & \text { Wrong } \\ & \mathrm{n}(\%) & \mathrm{n}(\%)\end{array}$

\section{Influenza : nature, symptoms and transmission ( $\mathrm{n}=6$ items)}

Influenza, caused by a virus can be a serious disease that can lead to hospitalization and sometimes even death

Anyone can't get very sick from flu including people who are healthy

$1 \quad 498(88.9 \%) \quad 62(11.1 \%)$

The signs and symptoms of influenza include fever, headache, sore throat, pain and aches

You can get flu from patients and coworkers who are sick with flu

If you become sick with flu, you can spread it to others even if you don't feel sick

Symptoms typically appear 8 to 10 days after a person is exposed to influenza

\section{Influenza vaccine safety and importance ( $\mathrm{n}=5$ items)}

The seasonal vaccine protects against the most common influenza viruses including $\mathrm{H} 1 \mathrm{~N} 1$

Flu vaccine cannot cause flu

$2361(64.5 \%)$

199(35.5\%)

Flu vaccines are safe, serious problems from a flu vaccine are very rare $3 \quad 497(88.8 \%) \quad 63(11.2 \%)$

$\mathrm{MOPH}$ recommends that $\mathrm{HCWs}$ receive influenza vaccine

$4 \quad 518(92.5 \%) \quad 42(7.5 \%)$

By getting vaccinated, you help protect yourself, your family, and your patients.

5

$517(92.3 \%) \quad 43(7.7 \%)$

\section{Influenza vaccine target groups ( $\mathrm{n}=4$ items 2$)$}

Vaccination to prevent flu is particularly important for people who are at high risk of developing serious flu complications Pregnant women and people with certain chronic health conditions can get $\quad 2 \quad 382(68.2 \%) \quad 178(31.8 \%)$
a flu shot

Children younger than 6 months of age are too young to get a flu should not get flu shot

$1 \quad 540(96.4 \%) \quad 20(3.6 \%)$

When vaccine supply is limited, vaccination efforts should be prioritized to: 4

Children aged 6 months through 4 years (59 months);

4a $\quad 224(40 \%) \quad 336(60 \%)$

People with chronic diseases pulmonary, cardiovascular, renal, hepatic, neurologic

$4 b \quad 463(82.7 \%) \quad 97(17.3 \%)$

People who are immunosuppressed 4c $\quad 408(72.9 \%) \quad 152(27.1 \%)$

Pregnant woman

$4 d \quad 199(35.5 \%) \quad 361(64.5 \%)$

Health care personnel;

4 e $\quad 430(76.8 \%)$

$130(23.2 \%)$

Administration and storage of vaccine ( $n=3$ items) 


\begin{tabular}{|c|c|c|c|}
\hline $\begin{array}{l}\text { A trivalent flu shot made using an adjuvant is approved for administration } \\
\text { for people } \geq 65 \text { y of age and older. }\end{array}$ & 1 & $372(66.4 \%)$ & 188(33.6\%) \\
\hline Antibodies develop in the body about 2 weeks after influenza vaccination. & 2 & $465(83.0 \%)$ & $95(17 \%)$ \\
\hline Influenza vaccine should be stored at 2 to $8 \circ \mathrm{C}$ & 3 & $517(92.3 \%)$ & $43(7.7 \%)$ \\
\hline \multicolumn{4}{|l|}{ Timing of vaccination ( $\mathrm{n}=3$ items) } \\
\hline $\begin{array}{l}\text { Vaccination should occur before onset of influenza activity in the } \\
\text { community. }\end{array}$ & 1 & $545(97.3 \%)$ & $15(2.7 \%)$ \\
\hline $\begin{array}{l}\text { Vaccination should continue to be offered as long as influenza viruses are } \\
\text { circulating }\end{array}$ & 2 & $427(76.3 \%)$ & $133(23.8 \%)$ \\
\hline $\begin{array}{l}\text { Annual vaccination is needed to get the "optimal" or best protection against } \\
\text { flu }\end{array}$ & 3 & $523(93.4 \%)$ & $37(6.6 \%)$ \\
\hline \multicolumn{4}{|l|}{ COVID-19 and influenza ( $n=3$ items) } \\
\hline Flu vaccine didn't protect against COVID-19 & 1 & $481(85.9 \%)$ & $79(14.1 \%)$ \\
\hline Both viruses influenza and COVID-19 are transmitted by respiratory droplets & 2 & $554(98.9 \%)$ & $6(1.1 \%)$ \\
\hline $\begin{array}{l}\text { COVID-19, and influenza are vastly different pathogens, but there are } \\
\text { important areas of overlap }\end{array}$ & 3 & $459(82 \%)$ & 101(18\%) \\
\hline
\end{tabular}


Table 4 HCWs' attitudes toward influenza vaccination items $(N=560)$

\begin{tabular}{lllll}
$\begin{array}{l}\text { Strongly } \\
\text { disagree }\end{array}$ & Disagree & Neutral & Agree & $\begin{array}{l}\text { Strongly } \\
\text { agree }\end{array}$ \\
\hline $\mathrm{n}(\%)$ & $\mathrm{n}(\%)$ & $\mathrm{n}(\%)$ & $\mathrm{n}(\%)$ & $\mathrm{n}(\%)$
\end{tabular}

General attitudes towards influenza and vaccination $(\mathrm{N}=560)$

I think it is not compulsory for HCW $\quad 241(43 \%) \quad 174(31.1 \%) \quad 55(9.8 \%) \quad 70(12.5 \%) \quad 20(3.6 \%)$

to get vaccinated for Influenza

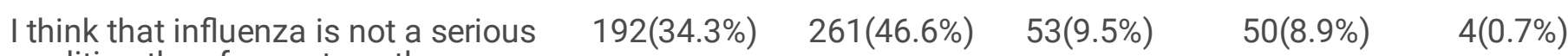
condition therefore not worth

vaccinating against

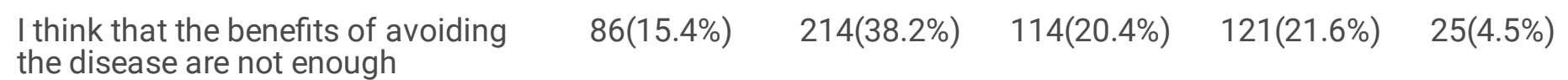

$\begin{array}{lllll}\text { I think vaccines weaken or overload } & 141(25.2 \%) & 298(53.2 \%) & 70(12.5 \%) & 43(7.7 \%)\end{array}$

the immune system

$\begin{array}{lllll}\text { It is better for me to develop natural } \quad 74(13.2 \%) & 240(42.9 \%) & 118(21.1 \%) & 111(19.8 \%) & 17(3 \%)\end{array}$

immunity by getting sick rather than

to get a vaccine

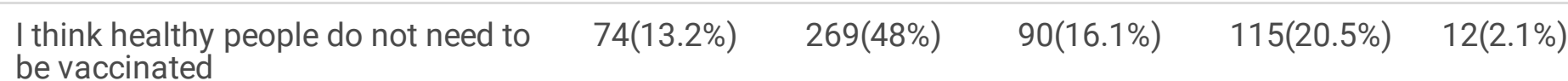

$\begin{array}{lllll}\text { I consider that allergies are on the } \quad 69(12.3 \%) & 282(50.4 \%) & 137(24.5 \%) & 69(12.3 \%) & 3(0.5 \%)\end{array}$

rise because of vaccinations

I think that frequency of adverse

reactions to influenza vaccines is

$17(3 \%) \quad 122(21.8 \%) \quad 197(35.2 \%) \quad 206(36.8 \%) \quad 18(3.2 \%)$

underestimated

$\begin{array}{lllll}\text { Vaccines are among the safest and } \quad 10(1.8 \%) & 46(8.2 \%) & 145(25.9 \%) & 324(57.9 \%) & 35(6.3 \%)\end{array}$

most tested medicinal products

HCWs Attitudes towards health facility, government and society $(\mathrm{N}=560)$

$\begin{array}{lllll}\text { I think that health care facilities } \quad 54(9.6 \%) & 136(24.3 \%) & 122(21.8 \%) & 217(38.8 \%) & 31(5.5 \%)\end{array}$

should ensure availability of

influenza vaccine at their institutions

$\begin{aligned} & \text { I think that the government should } \\ & \text { finance the vaccine for all }\end{aligned} \quad 7(1.3 \%) \quad 27(4.8 \%) \quad 47(8.4 \%) \quad 202(36.1 \%) \quad 277(49.5 \%)$

I think Lebanese society has more

important problems than influenza

I think vaccine policy in Lebanon is $\quad 9(1.6 \%) \quad 56(10 \%) \quad 141(25.2 \%) \quad 217(38.8 \%) \quad 137(24.5 \%)$

influenced by financial profits of

pharmaceutical companies

I think that vaccine information

provided by health authorities and

$15(2.7 \%) \quad 66(11.8 \%) \quad 185(33 \%) \quad 268(47.9 \%) \quad 26(4.6 \%)$

scientific societies is reliable 


\section{Table 5: Practices items of HCWs}

$\begin{array}{lll}\text { Never } & \text { Occasionally } & \text { Always } \\ n(\%) & n(\%) & n(\%)\end{array}$

\section{Vaccine promotion and Advertising}

\begin{tabular}{|c|c|c|c|}
\hline I encourage my patients to get flu vaccine & $36(6.4 \%)$ & $213(38 \%)$ & $311(55.5 \%)$ \\
\hline I encourage my colleagues and the office staff to get flu vaccine & $46(8.2 \%)$ & $194(34.6 \%)$ & $320(57.1 \%)$ \\
\hline $\begin{array}{l}\text { I encourage my family members who need to be vaccinated to } \\
\text { get vaccinated }\end{array}$ & $43(7.7 \%)$ & $182(32.5 \%)$ & $335(59.8 \%)$ \\
\hline $\begin{array}{l}\text { I encourage HCWs to get flu vaccine to minimize sick days, loss } \\
\text { of productivity and to ensure patient safety }\end{array}$ & $46(8.2 \%)$ & $167(29.8 \%)$ & $347(62 \%)$ \\
\hline $\begin{array}{l}\text { I encourage HCWs to get flu vaccine to avoid dual infection by } \\
\text { COVID- } 19\end{array}$ & $41(7.3 \%)$ & $177(31.6 \%)$ & $342(61.1 \%)$ \\
\hline $\begin{array}{l}\text { I encourage HCWs to get vaccinated to set an example to other } \\
\text { workers }\end{array}$ & $62(11.1 \%)$ & $156(27.9 \%)$ & $342(61.1 \%)$ \\
\hline
\end{tabular}

\section{Addressing vaccine hesitance}

I communicate the importance of getting influenza vaccine during office/clinics visits

I communicate the importance of getting influenza vaccine by telephone or by email

I use brochures and posters in my clinic/office revealing the importance of vaccine

I send influenza vaccine reminder by text to my patient

I celebrate event related to vaccination (vaccination day.....)

\begin{tabular}{|lll|}
\hline $90(16.1 \%)$ & $243(43.4 \%)$ & $227(40.5 \%)$ \\
\hline $268(47.9 \%)$ & $193(34.5 \%)$ & $99(17.7 \%)$ \\
\hline $163(29.1 \%)$ & $264(47.1 \%)$ & $133(23.8 \%)$ \\
\hline $298(53.2 \%)$ & $165(29.5 \%)$ & $97(17.3 \%)$ \\
\hline $285(50.9 \%)$ & $184(32.9 \%)$ & $91(16.3 \%)$ \\
\hline
\end{tabular}

\section{Trainings and Workshops}

I participate in trainings related to influenza vaccine in the past

46(8.2\%)

147(26.3\%)

$367(65.5 \%)$

I encourage my staff (HCWs) to participate in trainings related to

$74(13.2 \%)$

$227(40.5 \%)$

$259(46.3 \%)$

influenza vaccine 
Table 6: Logistic regression results for influenza vaccine uptake

Influenza vaccine uptake

\begin{tabular}{|c|c|c|c|c|c|c|}
\hline & \multirow{2}{*}{$\begin{array}{l}\text { No } \\
n=111(\%)\end{array}$} & \multirow{2}{*}{$\begin{array}{l}\text { Yes } \\
n=449(\%)\end{array}$} & \multirow[t]{2}{*}{ p-value } & \multirow[t]{2}{*}{ ORa } & \multicolumn{2}{|c|}{$\mathrm{ORa}(\mathrm{Cl} 95 \%)$} \\
\hline & & & & & Lower & Upper \\
\hline Gender & & & 0.612 & & & \\
\hline Male & $28(18.4 \%)$ & $124(81.6 \%)$ & & & & \\
\hline Female & $83(20.3 \%)$ & $325(79.7 \%)$ & & & & \\
\hline Age (years) & & & 0.604 & & & \\
\hline$<30$ & $41(18.7 \%)$ & $178(81.3 \%)$ & & & & \\
\hline $30-49$ & $64(21.2 \%)$ & $238(78.8 \%)$ & & & & \\
\hline 50 and above & $6(15.4 \%)$ & $33(84.6 \%)$ & & & & \\
\hline Urbanicity & & & 0.489 & & & \\
\hline Urban & $59(18.8 \%)$ & $255(81.2 \%)$ & & & & \\
\hline Rural & $52(21.1 \%)$ & 194(78.9\%) & & & & \\
\hline Occupation & & & 0.273 & & & \\
\hline Physician & $12(14.8 \%)$ & $69(85.2 \%)$ & & & & \\
\hline Pharmacist & $12(16.2 \%)$ & $62(83.8 \%)$ & & & & \\
\hline Lab technician & $14(27.5 \%)$ & $37(72.5 \%)$ & & & & \\
\hline Nurses & $73(20.6 \%)$ & $281(79.4 \%)$ & & & & \\
\hline Social status & & & 0.041 & & & \\
\hline Married & $71(19.2 \%)$ & $299(80.8 \%)$ & Reference & & & \\
\hline Others (Single, divorced) & $40(21.1 \%)$ & $150(78.9 \%)$ & $0.042 *$ & 0.527 & 0.284 & 0.978 \\
\hline Perceived Health status & & & 0.370 & & & \\
\hline Good and Above & $97(20.5 \%)$ & $377(79.5 \%)$ & Reference & & & \\
\hline Fair and below & $14(16.3 \%)$ & 72(83.7\%) & $0.008 *$ & 3.117 & 1.345 & 7.220 \\
\hline Presence of comorbidities & & & 0.851 & 0.954 & 0.585 & 1.556 \\
\hline Yes & $26(19.3 \%)$ & $109(80.7 \%)$ & & & & \\
\hline No & $85(20 \%)$ & $340(80 \%)$ & & & & \\
\hline Type of the hospital & & & 0.118 & & & \\
\hline Private & $90(21.3 \%)$ & $332(78.7 \%)$ & & & & \\
\hline Public & $21(15.2 \%)$ & $117(84.8 \%)$ & & & & \\
\hline Health Coverage & & & 0.605 & & & \\
\hline
\end{tabular}




\begin{tabular}{|c|c|c|c|c|c|c|}
\hline Public & $94(19 . .2 \%)$ & $396(80.8 \%)$ & & & & \\
\hline Private & $7(24.1 \%)$ & $22(75.9 \%)$ & & & & \\
\hline None & $10(24.4 \%)$ & $31(75.9 \%)$ & & & & \\
\hline Years of experience & & & 0.926 & & & \\
\hline$<10$ years & $62(19.7 \%)$ & $253(80.3 \%)$ & & & & \\
\hline$>10$ years & $49(20 \%)$ & $196(80 \%)$ & & & & \\
\hline $\begin{array}{l}\text { Received influenza vaccine in the past } \\
\text { season }\end{array}$ & & & $<0.001^{*}$ & & & \\
\hline No & $100(9.9 \%)$ & $280(37.6 \%)$ & Reference & & & \\
\hline Yes & $11(6.1 \%)$ & 169(93.9\%) & $<0.001 *$ & 6.812 & 3.045 & 15.239 \\
\hline Knowledge about safety of vaccine & & & $<0.001^{*}$ & & & \\
\hline Poor Knowledge & $26(66.7 \%)$ & 13(33.3\%) & Reference & & & \\
\hline Moderate Knowledge & $28(31.1 \%)$ & $62(68.9 \%)$ & 0.175 & 2.205 & 0.703 & 6.913 \\
\hline Good Knowledge & $57(13.2 \%)$ & $374(86.8 \%)$ & $0.026 *$ & 3.305 & 1.155 & 9.457 \\
\hline General attitude toward vaccination & & & $<0.001^{*}$ & & & \\
\hline Negative & $30(60 \%)$ & $20(40 \%)$ & Reference & & & \\
\hline Neutral & $22(56.4 \%)$ & $17(43.6 \%)$ & 0.065 & 0.366 & 0.125 & 1.066 \\
\hline Positive & $59(12.5 \%)$ & $412(87.5 \%)$ & $0.047^{*}$ & 2.305 & 0.921 & 5.771 \\
\hline $\begin{array}{l}\text { Attitudes towards government and } \\
\text { society }\end{array}$ & & & 0.338 & & & \\
\hline Negative & $97(22.7 \%)$ & $331(77.3 \%)$ & & & & \\
\hline Neutral & $14(10.9 \%)$ & $115(89.1 \%)$ & & & & \\
\hline Positive & $0(0 \%)$ & $3(100 \%)$ & & & & \\
\hline Perceived benefits & & & $<0.001^{*}$ & & & \\
\hline Low & $37(56.1 \%)$ & $29(43.9 \%)$ & Reference & & & \\
\hline Moderate & $27(29 \%)$ & $66(71 \%)$ & $0.005^{*}$ & 3.433 & 1.466 & 8.040 \\
\hline High & $47(11.7 \%)$ & $354(88.3 \%)$ & $<0.001^{*}$ & 6.264 & 2.919 & 13.442 \\
\hline Perceived barriers & & & 0.001 & & & \\
\hline High & $29(53.7 \%)$ & $25(46.3 \%)$ & Reference & & & \\
\hline Moderate & $36(31.3 \%)$ & $79(68.7 \%)$ & 0.166 & 1.808 & 0.783 & 4.177 \\
\hline Low & $46(11.8 \%)$ & $345(88.2 \%)$ & $<0.001^{*}$ & 4.130 & 1.827 & 9.334 \\
\hline
\end{tabular}




\title{
Figures
}

\section{Perceived barriers by HCWs for influenza vaccination}

\author{
Due to needle fear I don't like to get vaccinated $\square 4.82 \%$ \\ I think that influenza vaccine is not effective $\square 7.86 \%$ \\ I think that there is insufficient staff to administer vaccine \\ I have concern about safety of the vaccine $18.21 \%$ \\ I have concern with side effects of getting vaccinated for influenza $23.57 \%$ \\ I think that Influenza vaccine is costly that's why not purchased $=24.29 \%$ \\ I am afraid that one of my patients may develop a severe adverse reaction. $=31.07 \%$

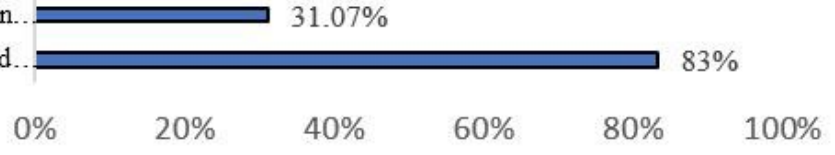

I think we will face a shortage in influenza vaccine due to the high demand

\section{Figure 1}

HCWs' perceived barriers for influenza vaccination

\section{Perceived benefits of influenza vaccination}

I think influenza vaccination of $\mathrm{HCW}$ can enhance patient safety

I think there is a heightened importance for influenza vaccination to minimize the viral reservoir in the population.

I think influenza vaccine can decrease in hospital visits and allow services to better cope with COVID-19 complications

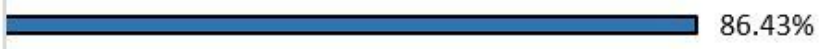

$86.43 \%$
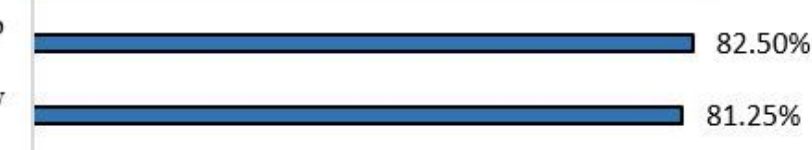

I think vaccines are important for my patients' health especially to

I think vaccination is cost-effective

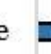

$39.29 \%$

$\begin{array}{llllll}0.00 \% & 20.00 \% & 40.00 \% & 60.00 \% & 80.00 \% & 100.00 \%\end{array}$

Figure 2

Perceived benefits of influenza vaccination according to HCWs 
HCWs vaccinated in the previous season (2019-2020) before COVID-19

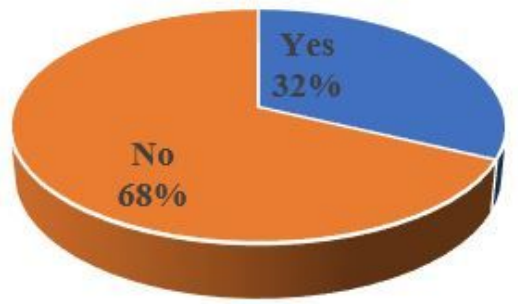

HCWs willingness to vaccinate in the era of COVID-19 (season 2020-2021)

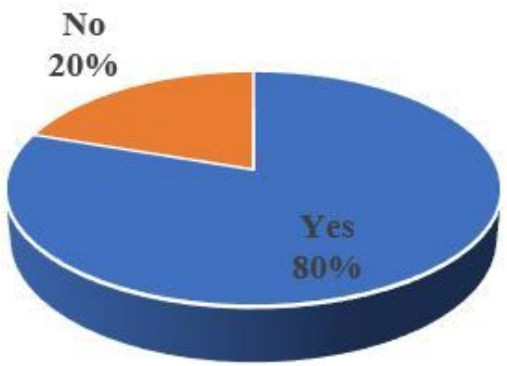

\section{Figure 3}

Influenza vaccination among HCWs before and during COVID-19 pandemic

\section{Supplementary Files}

This is a list of supplementary files associated with this preprint. Click to download.

- InfluenzaQuestionnaireKAPHCW.docx 